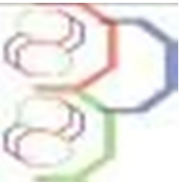

Journal of Applied Biosciences 154: 15862 - 15870

\title{
Effets du vermicompost à base d'ordures ménagères associées aux balles de riz sur les paramètres agronomiques du maïs (Zea mays L.)
}

\section{Arnauth Martinez GUEl1,2*, Ferdinand Gohi Bi ZRO1, Sidiky BAKAYOKO1, Fabrice Djè Bi TA1}

Université Jean Lorougnon Guédé, Unité de Formation et de Recherche en Agroforestérie, Département d'Agropédologie et SIG, BP 150 Daloa, Côte d'Ivoire.

2Université Nangui Abrogoua, Centre de Recherche en Ecologie, 08 BP 109 Abidjan 08, Côte d'Ivoire

Auteur correspondant : gueiarnauthmartinez@yahoo.fr Tel : (225) 48988857 / 05452992

Original submitted in on $3^{\text {rd }}$ July 2020. Published online at www.m.elewa.org/journals/ on $31^{\text {st }}$ October 2020 https://doi.org/10.35759/JABs.154.3

\section{RESUME}

Objectifs: Cette étude se veut de produire et de déterminer la valeur fertilisante du vermicompost, et mesurer les effets de ce vermicompost sur la croissance et le rendement du maïs.

Méthodologie et résultats : Les travaux ont d'abord consisté à élaborer un compost d'ordures ménagères puis son vermicompost associé par inoculation du ver de terre Eudrilus eugeniae (Kinb.), et ensuite à évaluer leurs effets sur la production du maïs à travers un essai conduit en milieu naturel au sein de l'Université Jean Lorougnon Guedé Daloa. Les traitements sont composés d'un témoin constitué d'un sol de jachère, d'un compost d'ordures ménagères associées aux balles de riz et du vermicompost correspondant. Les résultats ont révélé que le vermicompost a les teneurs les plus importantes en N, $P$ et $\mathrm{K}$, mais les plus faibles concentrations en $\mathrm{Cu}, \mathrm{Zn}$ et $\mathrm{Pb}$. Les plants de maïs croissent mieux sur le vermicompost par comparaison au compost et au témoin. De plus, le traitement de vermicompost a produit la biomasse racinaire $\left(45,625 \pm 1,812 \mathrm{~kg} \mathrm{ha}^{-1}\right)$, le nombre de grains par plant $\left(391,68 \pm 17,20 \mathrm{~kg} \cdot \mathrm{ha}^{-1}\right)$ et la biomasse totale des grains de maïs $\left(2608,969 \pm 50,914 \mathrm{~kg}_{\text {.ha-1 }}{ }^{-1}\right)$ les plus élevés.

Conclusions et application des résultats : Les travaux ont révélé l'importance de l'espèce Eudrilus eugeniae dans la formation d'un compost de bonne qualité chimique. Les paysans peuvent utiliser le vermicompost d'ordures ménagères associées aux balles de riz pour améliorer le rendement des cultures de maïs.

Mots clés : Vermicompost d'ordures ménagères, Balles de riz, maïs, croissance, rendement.

\begin{abstract}
Objectives: This study aims to produce and determine the fertilizing value of vermicompost, and to measure the effects of this vermicompost on the growth and yield of corn.

Methods and results: The work consisted first of all in developing a household waste compost then its associated vermicompost by inoculation of the earthworm Eudrilus eugeniae (Kinb.), and then in evaluating their effects on the production of maize through a trial conducted in the natural environment in Jean Lorougnon Guedé University of Daloa. The treatments consisted of a control consisting of fallow soil, household waste compost associated with rice husks and the corresponding vermicompost. The results revealed that vermicompost has the highest levels of $\mathrm{N}, \mathrm{P}$ and $\mathrm{K}$, but the lowest concentrations of $\mathrm{Cu}, \mathrm{Zn}$
\end{abstract}


and $\mathrm{Pb}$. Corn plants grow better on vermicompost compared to compost and control. In addition, the vermicompost treatment produced root biomass $\left(45.625 \pm 1.812 \mathrm{~kg}^{-h^{-1}}\right)$, the number of grains per plant $\left(391.68 \pm 17.20 \mathrm{~kg} \mathrm{ha}^{-1}\right)$ and the total biomass of grain $\left(2608.969 \pm 50.914 \mathrm{~kg} \cdot \mathrm{ha}^{-1}\right)$ the highest.

Conclusion and application of findings: The work revealed the importance of the species Eudrilus eugeniae in the formation of a compost of good chemical quality. Farmers can use household vermicompost associated with rice hulls to improve the yield of maize crops.

Keywords: Household waste vermicompost, rice husks, maize, growth, yield.

\section{INTRODUCTION}

La Côte d'Ivoire, à l'instar des pays en voie de développement, est caractérisée par une croissance démographique élevée au fil des années. La population qui vit majoritairement en milieu rural $(58,9 \%)$ a plus que triplée en l'espace de 39 ans, passant ainsi de 6709600 habitants en 1975 à 22671331 habitants en 2014 (RGPH, 2014). Cette croissance démographique a pour conséquence une forte pression anthropique sur les terres arables, entrainant à son tour la baisse de la productivité agricole due à la détérioration de la qualité biologique, physique et chimique des sols (Guéi et al., 2019 ; Tondoh et al., 2019). II est donc impératif de recourir à la fertilisation qui constitue la technique la plus appropriée et rapide pour apporter aux plantes les compléments indispensables. A cet effet, la fertilisation minérale et organique ont été adoptées pour satisfaire les besoins nutritifs des plantes en vue de leur assurer une croissance et un développement optimal (Coulibaly et al., 2018; Guéi et al., 2020). Mais l'utilisation intensive des engrais chimiques de synthèse aux dépens de la fumure organique augmente certes, le rendement immédiat, mais impacte négativement le sol, l'environnement et la santé humaine (FAO, 2015). En outre, ces engrais sont de plus en plus chers pour les petits paysans aux ressources financières limitées. II existe pourtant des voies pour sortir de cette impasse qui révisent les paradigmes régissant la gestion respectueuse de l'environnement de la fertilité des sols. L'une d'entre elles consiste à valoriser l'utilité des services écosystémiques délivrés par les populations de vers de terre qui sont des "ingénieurs de l'écosystèmes" (Jones et al., 1994). Dans cette catégorie, se trouve le vermicompostage et son utilisation comme amendement pour une production agricole durable. De nombreuses études ont démontré l'importance du vermicompost dans l'amélioration de la production agricole (Coulibaly et al., 2016; Chaichi et al., 2017 ; Rekha et al., 2018 ; Hien et al., 2018). Le vermicompost est un matériau stable issu de l'ingestion et de la digestion des déchets organiques par les vers de terres détritivores, qui améliore les propriétés physico-chimiques du sol (Suthar, 2007). Le vermicompost contient moins de métaux lourds que le compost grâce à l'action des vers de terre (Coulibaly et al., 2018). Cependant, très peu d'études existent sur l'aptitude du ver de terre détritivore Eudrilus eugeniae (Kinb.) dans l'élaboration de vermicompost à partir des ordures ménagères en vue d'une optimisation de la production du maïs, la céréale la plus cultivée au monde. Le maïs constitue la base du régime alimentaire des populations rurales dans les régions tropicales et subtropicales (FAO, 2020; Haro et al., 2020), il apparait donc nécessaire d'améliorer la fertilité du sol par l'utilisation des engrais organiques pour soutenir sa production. La présente étude se propose d'évaluer l'impact du vermicompost d'ordures ménagères associées aux balles de riz sur la croissance et le rendement du maïs. De façon spécifique, il s'agit de (1) produire le vermicompost et de déterminer sa valeur fertilisante et (2) mesurer les effets de ce vermicompost sur la croissance et le rendement du maïs. 


\section{MATERIELS ET METHODES}

Site d'étude: Cette étude a été réalisée au sein de l'Université Jean Lorougnon Guédé, dans la ville de Daloa, région du Haut-Sassandra située au CentreOuest de la Côte d'Ivoire $\left(6^{\circ}\right.$ et $7^{\circ}$ de Latitude Nord et, $7^{\circ}$ et $8^{\circ}$ de Longitude Ouest). Cette région a une superficie de $15.200 \mathrm{~km}^{2}$ pour une population estimée à 1430960 habitants (RGPH, 2014). La parcelle expérimentale est située entre $6^{\circ} 54^{\prime}$ de Latitude Nord et $6^{\circ} 26^{\prime}$ de Longitude Ouest. Le régime climatique est celui du domaine Guinéen caractérisé par un régime équatorial et subéquatorial à deux maxima pluviométriques. La pluviométrie moyenne annuelle oscillant entre 1200 et $1600 \mathrm{~mm}$. Le mois de juin représente le pic de la grande saison pluvieuse et celui de septembre le pic de la petite saison des pluies. Ces deux maximas sont séparés par un ou deux mois plus ou moins pluvieux (Brou, 2005). Les études pédologiques réalisées dans la zone révèlent que les sols y sont en général ferralitiques moyennement lessivés (ou désaturés) (Dabin et al., 1960).

Production de compost et vermicompost: Deux fosses de dimension $2 \times 1 \times 1 \mathrm{~m}$ (longueurs $\mathrm{x}$ largeurs $\mathrm{x}$ profondeurs) ont été ouvertes dans un endroit ombragé non inondable. Dans chaque compostière, la fraction compostable a été disposée par couches successives alternées de tontes de gazon, d'ordures ménagères et de balles de riz jusqu'à l'obtention d'une hauteur d'un mètre. Les ordures ménagères ont été collectées dans différents quartiers de la ville de Daloa. Les balles de riz ont été collectées au quartier Abattoir 2, les épluchures de manioc, de banane et d'igname ont été collectées au quartier Huberson et les autres ordures ménagères ont été fournies par le service de restauration des étudiants de l'université Jean Lorougnon Guédé. Les ordures collectées ont été triées pour les débarrasser de matériels non biologiques et non fermentescibles. Le tas d'ordures a été arrosé puis chaque fosse a été couverte d'une bâche noire. Au bout d'une période de 60 jours de précompostage, après que les gaz volatiles toxiques aient évacués (Coulibaly et al., 2016), le compost de chaque fosse a été divisé en deux parties dont une partie a été consacrée au compostage ordinaire et l'autre au vermicompostage. Les individus de l'espèce Eudrilus eugeniae récoltés sous des décharges d'ordures ménagères ont été inoculés le matin dans chaque demie fosse à une densité de 3000 individus par mètre cube, ce qui correspond à un taux de 3 vers de terre par litre de compost (Deka et al., 2011). Après maturation, lorsque la température s'est stabilisée au bout de 3 mois et demi (105 jours), les différents types de composts ont été prélevés des fosses et séchés à l'air libre durant deux semaines. Les composts ont été tamisés à travers des mailles de $2 \mathrm{~mm}$. Pour un type de compost donné, 9 prélèvements à différents endroits ont été mélangés pour obtenir un échantillon composite; 3 échantillons composites ont ainsi été constitués pour chaque type de compost. Trois aliquotes de $0,5 \mathrm{~kg}$ de chaque type de composts ont été constitués. Les échantillons constitués ont été envoyés pour les analyses chimiques au Laboratoire d'Analyse des Sols et des Végétaux (LAVESO) de l'Ecole Supérieure d'Agronomie sise à l'Institut National polytechnique Félix Houphouët-Boigny (INP-HB). Le $\mathrm{pH}_{\text {eau }}$ a été mesuré dans le surnageant du mélange compost-eau dans un rapport 1/2,5 à l'aide d'un $\mathrm{pH}$ mètre à électrodes combinés. La matière organique et le carbone ont été déterminés par la Méthode Walkley \& Black (1934), l' azote total par la méthode de Kjeldahl à partir de la digestion du compost dans une solution de $\mathrm{H}_{2} \mathrm{SO}_{4}$ concentré en présence de catalyseur $\left(\mathrm{K}_{2} \mathrm{SO}_{4}\right)$. Le phosphore assimilable a été extrait par la méthode d'Olsen-Dabin (Murphy \& Riley, 1962). Le potassium a été déterminé par lavage alcoolique suivi d'une distillation Kjeldahl (Anderson \& Ingram, 1993). Les teneurs en éléments métalliques $(\mathrm{Zn}$, $\mathrm{Cu}$ et $\mathrm{Pb})$ ont été déterminées par spectrophotométrie d'absorption atomique à flamme après minéralisation des échantillons à l'eau.

Dispositif expérimental, traitements et conduite de l'essai : Le dispositif expérimental adopté est un bloc complètement randomisé. II comporte 4 blocs avec 3 traitements sur une superficie totale $62,4 \mathrm{~m}^{2}$, soit un total de 12 parcelles élémentaires. Chacune des parcelles élémentaires couvre une superficie de $2,4 \mathrm{~m}^{2}$ $(1,20 \mathrm{~m} \times 2 \mathrm{~m}$ ) espacée par une allée de $1 \mathrm{~m}$ (figure $1 \mathrm{~A})$. Le compost et le vermicompost ont été enfouis dans le sol sur chacune des parcelles élémentaires sous forme de fumure de fond en dose unique deux semaines avant le semis à chaque cycle de production du maïs. Au total, les composts ont été appliqués sur une culture de maïs pendant deux cycles successifs, et la dose appliquée qui est égale à 40 tha $^{-1}$ correspond à la dose optimale de production déterminée par Guéi et al. (2020) pour le compost de bouse de bovins. Les 2 traitements qui ont consistés en un apport de composts sont comparés à un témoin (sol sans amendement) :

T0 : Témoin (sol sans amendement), C.OM + br: Sol + 40 t.ha $^{-1}$ de compost d'ordures ménagères mélangées aux balles de riz, 
VC.OM + br : Sol + 40 t.ha-1 de vermicompost d'ordures ménagères mélangées aux balles de riz.

Le semis du maïs a été réalisé directement dans des poquets de faible profondeur, environ $3 \mathrm{~cm}$. Deux à trois grains ont été placés par poquet. Le maïs est semé en lignes distantes de $80 \mathrm{~cm}$ tandis que les poquets sont situés à $20 \mathrm{~cm}$ l'un de l'autre. Le démariage s'est déroulé deux semaines après semis pour ne laisser qu'un seul pied par poquet, ceci correspond à une densité après démariage de 28846 plants à l'hectare. Chaque parcelle élémentaire a bénéficié de deux sarclages : le premier a été effectué deux semaines après semis et le second deux semaines après le premier. Sur l'ensemble des 12 parcelles élémentaires, les mesures des paramètres agronomiques ont été effectuées d'une manière identique sur un total de 10 plants par traitement, en raison de 5 plants par ligne (figure 1B).

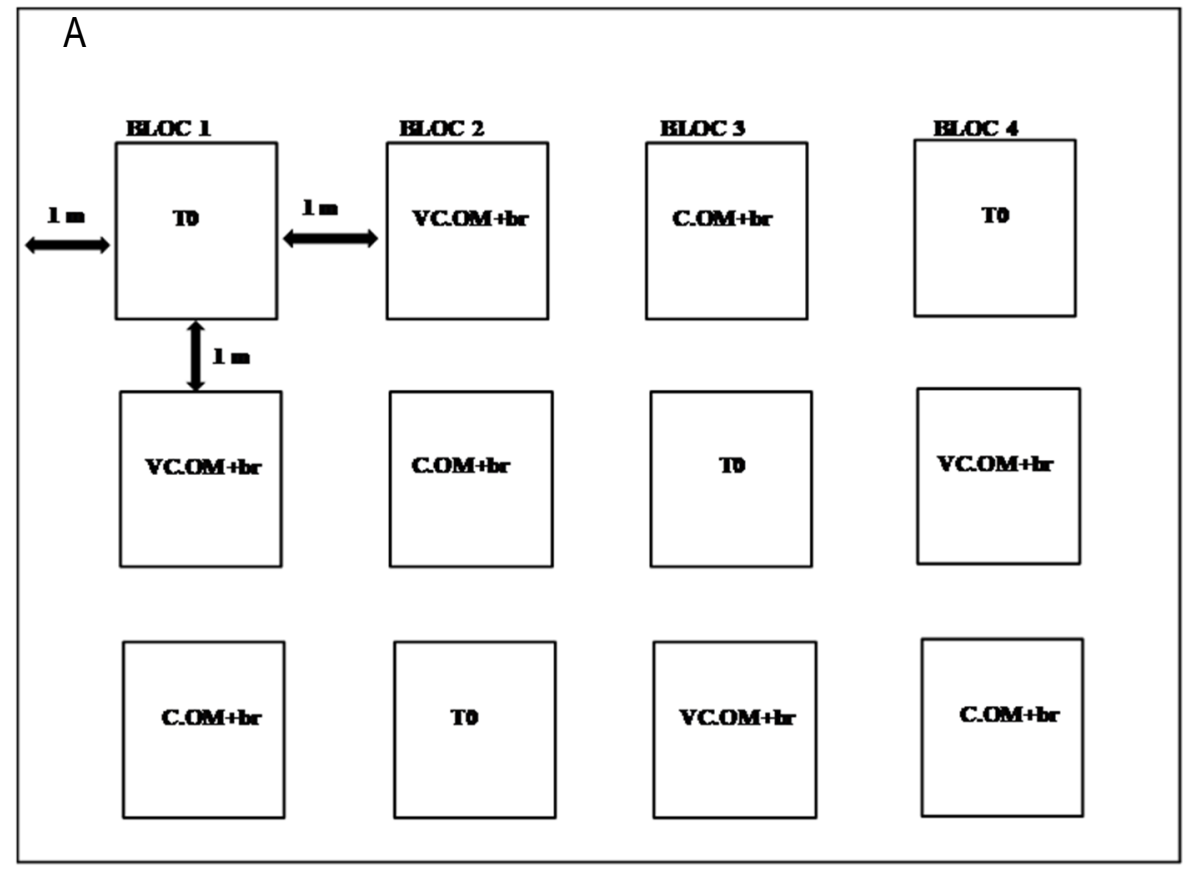

B

$2 \mathrm{~m}$

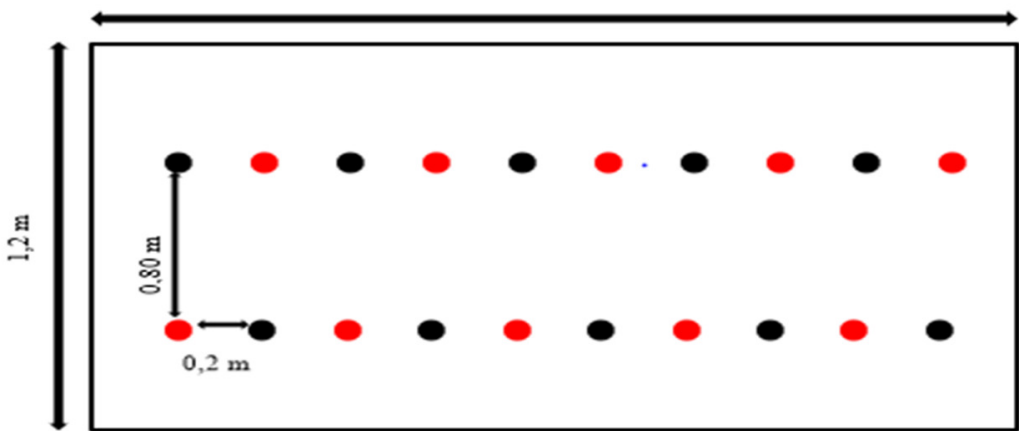

Plante ayant fait l'objet de mesure

Plante n'ayant pas fait l'objet de mesure

Figure 1: Protocole d'expérimentation: (A) Dispositif expérimental et (B) disposition des plants sur une parcelle élémentaire. C.OM+br: Compost d'ordures ménagères associées aux balles de riz, VC.OM+br: Vermicompost d'ordures ménagères associées aux balles de riz, T0 : Traitement témoin 
Quinze jours après semis, dix (10) pieds de maïs de chaque parcelle élémentaire ont été sélectionnés pour les mesures des paramètres agronomiques. En effet, quatre (4) paramètres de croissance ont été mesurés par pied. II s'agit du nombre de feuilles; longueur de la plus grande feuille $(\mathrm{cm})$ et la hauteur des plants $(\mathrm{cm})$ (du collet à l'apex) mesurés à l'aide d'un mètre-ruban, et du diamètre au collet $(\mathrm{cm})$ évalué à l'aide du pied à coulisse. Ces paramètres de croissance ont été mesurés sur des pas de temps de 15 jours après semis jusqu'au $75^{\mathrm{e}}$ jour. A la récolte, les plants de maïs ont été prélevés entièrement (parties aériennes et racines), étiquetés et transportés au laboratoire où ils ont été séchés à l'étuve à $60^{\circ} \mathrm{C}$, pendant $72 \mathrm{~h}$. Pour chaque plante, les racines et la partie épigée ont été séparées et pesées en vue de déterminer respectivement la biomasse racinaire et la biomasse épigée. Ensuite les

\section{RESULTATS}

Caractéristiques chimiques des composts et du sol témoin : Les caractéristiques chimiques des composts et du sol de jachère avant la mise en place des essais agronomiques ont été déterminées. Ces caractéristiques sont présentées dans le tableau 1. Ces résultats montrent que le $\mathrm{pH}$ du compost d'ordures ménagères est neutre $(7,5 \pm 0,05)$ tandis que ceux du vermicompost $(7,37 \pm 0,01)$ et du sol de jachère $(7,71$ $\pm 0,14)$ sont basiques. La matière organique est plus épis ont été égrenés, puis les grains ont été comptés et pesés. La masse des différentes parties a été rapportée à la tonne à l'hectare. Trois échantillons composites de sol ont été prélevés à la profondeur $0-20 \mathrm{~cm}$ pour la réalisation des analyses chimiques, notamment le $\mathrm{pH}$ eau, les matières organiques, N, P et K.

Analyses statistiques: Les moyennes ont été soumises à une analyse de variance à un facteur après la vérification de la normalité de la distribution (Test de Shapiro-Wilk) et de l'homogénéité des variances (test de Levene). Ensuite, quand il y a une différence significative entre les moyennes, le test post Anova LSD de Fischer au seuil de $5 \%$ a été utilisé pour faire la comparaison deux à deux et en déduire les groupes homogènes. Ces analyses statistiques ont été réalisées à l'aide du logiciel Statistica version 7.1 (Statsoft, Tulsa, USA).

importante dans les composts. Pour ce qui concerne les teneurs en éléments majeurs, à l'exception du phosphore assimilable qui est élevé dans le sol de jachère, le $\mathrm{N}$ et $\mathrm{K}$ ont de grandes teneurs dans le compost d'ordures ménagères (C.OM) et son vermicompost associé (VC.OM). Les teneurs en éléments traces métalliques (ETM) du vermicompost $(\mathrm{Zn}: 96,60 \pm 0,92 ; \mathrm{Cu}: 13,93 \pm 0,24 ; \mathrm{Pb}: 21,33 \pm$ $0,66)$ sont plus faibles que celles du compost.

Tableau 1 : Caractéristiques chimiques des composts et du sol témoin

\begin{tabular}{|c|c|c|c|c|c|c|c|c|c|}
\hline \multirow[t]{3}{*}{ Traitements } & \multicolumn{9}{|c|}{ Eléments chimiques des substrats } \\
\hline & \multirow{2}{*}{$\begin{array}{l}\text { Acidité } \\
\text { pH eau }\end{array}$} & \multicolumn{2}{|c|}{$\begin{array}{c}\text { Matières } \\
\text { organiques \% }\end{array}$} & \multicolumn{3}{|c|}{ Eléments majeurs } & \multicolumn{3}{|c|}{$\begin{array}{c}\text { Eléments traces } \\
\text { métalliques }(\mathrm{mg} / \mathrm{kg})\end{array}$} \\
\hline & & C & MO & $\begin{array}{c}\mathbf{N} \\
\left(g^{\prime} \cdot \mathrm{kg}^{-1}\right)\end{array}$ & $\begin{array}{c}\text { P. ass } \\
\left(\mathrm{mg}^{\left.-k^{-1}\right)}\right.\end{array}$ & $\begin{array}{c}\mathrm{K}+ \\
\left(\text { cmolc.kg-1) }^{-1}\right)\end{array}$ & $\mathrm{Zn}$ & $\mathrm{Cu}$ & $\mathrm{Pb}$ \\
\hline C.OM & $\begin{array}{l}7,5 \pm \\
0,05\end{array}$ & $\begin{array}{l}13,39 \\
\pm 0,2\end{array}$ & $\begin{array}{c}7,79 \pm \\
0,01\end{array}$ & $\begin{array}{c}0,70 \pm \\
0,20\end{array}$ & $\begin{array}{c}24,70 \pm \\
0,61\end{array}$ & $2,93 \pm 0,07$ & $\begin{array}{c}121,33 \pm \\
0,88\end{array}$ & $\begin{array}{c}17,33 \pm \\
0,09\end{array}$ & $\begin{array}{c}25,03 \pm \\
0,09\end{array}$ \\
\hline VC. OM+br & $\begin{array}{c}7,37 \pm \\
0,01\end{array}$ & $\begin{array}{l}14,74 \\
\pm 0,7\end{array}$ & $\begin{array}{c}8,30 \pm \\
0,17\end{array}$ & $\begin{array}{c}0,93 \pm \\
0,09\end{array}$ & $\begin{array}{c}28,43 \pm \\
0,59\end{array}$ & $3,27 \pm 0,12$ & $\begin{array}{c}96,60 \pm \\
0,92\end{array}$ & $\begin{array}{c}13,93 \pm \\
0,24\end{array}$ & $\begin{array}{c}21,33 \pm \\
0,66\end{array}$ \\
\hline $\begin{array}{l}\text { Sol de } \\
\text { jachère }\end{array}$ & $\begin{array}{c}7,71 \pm \\
0,14\end{array}$ & $\begin{array}{c}1,69 \pm \\
1,47\end{array}$ & $\begin{array}{c}2,90 \pm \\
2,54\end{array}$ & $\begin{array}{c}0,13 \pm \\
0,06\end{array}$ & $\begin{array}{c}56,00 \pm \\
4,58\end{array}$ & $0,59 \pm 0,05$ & ND & ND & ND \\
\hline
\end{tabular}

C.OM +br : Compost d'Ordures Ménagères associées aux balles de riz, VC.OM+br : Vermicompost d'Ordures Ménagères associées aux balles de riz ; MO : Matières organiques ; ND : Non déterminé.

Effets sur les paramètres de croissance du maïs : Quel que soit le traitement considéré, le nombre de feuilles des plants par hectare a augmenté régulièrement avec le temps (figure $2 \mathrm{~A}$ ). Le nombre de feuilles a varié de $2365,86 \pm 96,56$ à $5725,07 \pm 43,79$ environ avec les composts. Par contre, dans le traitement témoin, le nombre de feuilles qui est de
$23592,92 \pm 29,87$ feuilles.ha-1 à 15 jours après semis (JAS), passe à 56944,44 \pm 163,3 feuilles.ha-1à 75 JAS. Toutefois, les comparaisons inter traitements à chaque pas de temps de mesure montrent que le vermicompost d'ordures ménagères associées aux balles de riz (VC.OM+ br) n'a pas significativement (Anova, $p>$ $0,05)$ induit une augmentation du nombre de feuilles 
par comparaison aux composts et sol témoin. Par contre, à partir du 30ème jusqu' au 75ème jour de croissance (figure $2 \mathrm{~B}$ ), les longueurs des feuilles sont significativement différentes entre les traitements (Anova 1, $p<0,05$ ). En effet, les pieds de maïs cultivés sur le vermicompost présentent les plus longues feuilles au 30 ème jour $(63,6 \pm 2,94 \mathrm{~cm})$, $45^{\text {ème }}$ jour $(85,6$

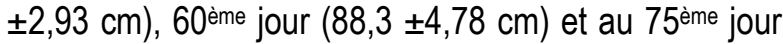
$(95,1 \pm 4,80 \mathrm{~cm})$. Le vermicompost est suivi par le compost d'ordures ménagères associées aux balles de riz (30 JAS :59,9 $\pm 3,24 \mathrm{~cm} ; 45$ JAS : $82,9 \pm 2,42 \mathrm{~cm}$; 60 JAS : $84,9 \pm 2,83 \mathrm{~cm}$ et $75 \mathrm{JAS}: 90,7 \pm 4,80 \mathrm{~cm})$. Le témoin a donné les plus courtes feuilles. De manière similaire à la longueur de la feuille, les pieds de maïs présentent des diamètres au collet (figure $2 \mathrm{C}$ ) plus élevés sur le vermicompost ( $30 \mathrm{JAS}: 5,80 \pm 1,80 \mathrm{~cm}$; 45 JAS : 6,05 $\pm 0,17 \mathrm{~cm} ; 60$ JAS : 6,60 $0,19 \mathrm{~cm} ; 75$ JAS : 6,64 $\pm 0,20 \mathrm{~cm})$. Les résultats de l'analyse de variance à un facteur révèlent des différences significatives entre les trois traitements à $30 \mathrm{JAS}$ ( $p=$ $0,018), 45$ JAS $(p<0,001), 60$ JAS $(p<0,001)$ et 75 JAS $(p<0,001)$. Deux groupes de plants se dégagent à partir du $45 \mathrm{JAS}$ (figure 2D). D'une part, il y a le groupe des composts qui portent les plants les plus hauts, et d'autre part les plants semés sur sol témoin qui sont les plus courts (Anova 1, $p<0,05$ ).
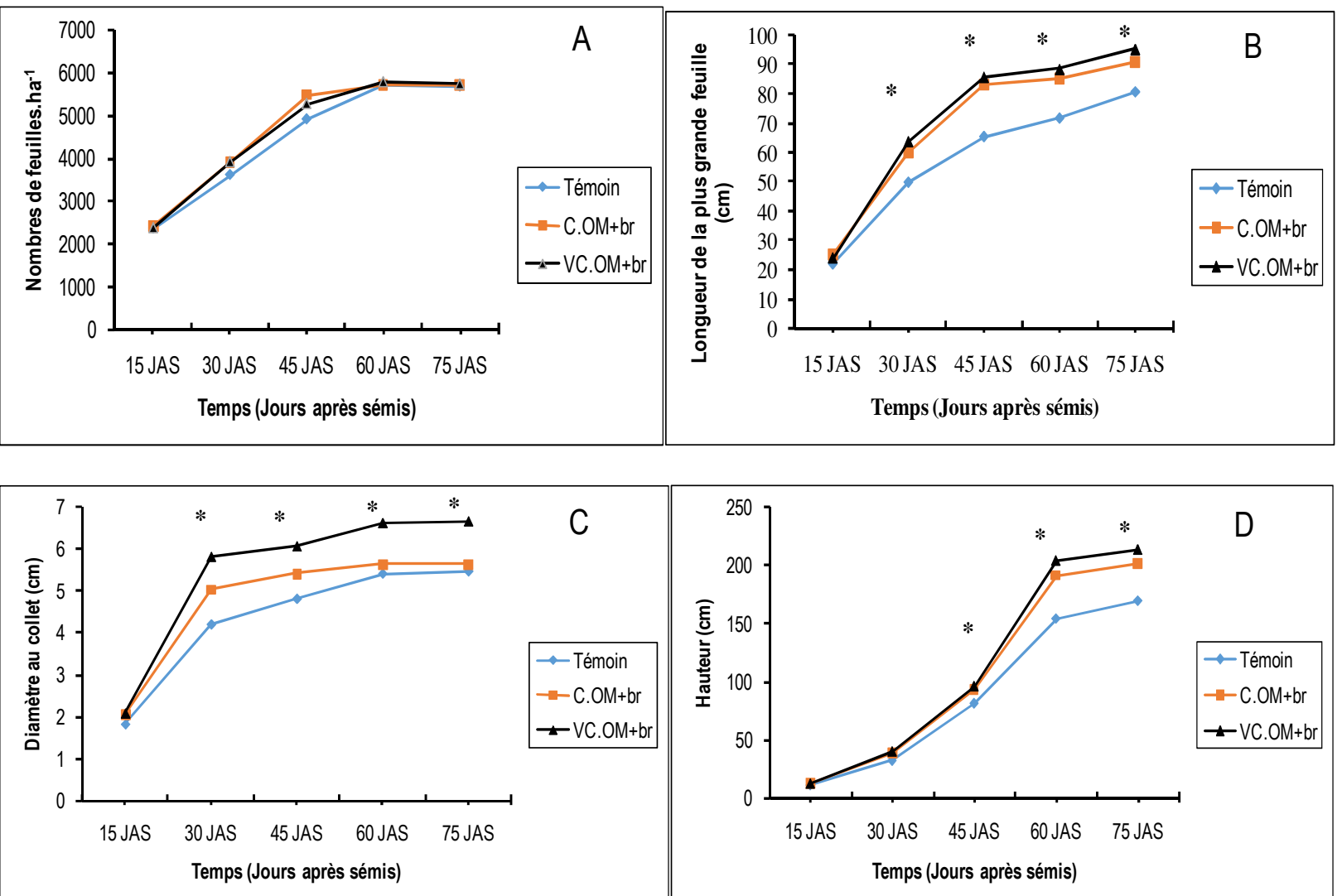

Figure 2 : Evolution des paramètres de croissance du maïs en fonction du temps et des traitements : (A) Nombre de feuilles, (B) Longueur de la plus grande feuille, (C) Diamètre au collet et (D) Hauteur. C.OM+br : Compost d'ordures ménagères associées aux balles de riz, $\mathrm{VC} . \mathrm{OM}+\mathrm{br}$ : Vermicompost d'ordures ménagères associées aux balles de riz, Témoin : Sol de jachère. Pour un pas de temps donné, l'astérisque indique que les valeurs sont significativement différentes (Anova 1, $p=0,05)$.

Effets sur les paramètres de production et le rendement du maïs: Les biomasses épigées des pieds de maïs issus des traitements de vermicompost et compost sont statistiquement identiques, mais plus grandes que celle obtenue avec le traitement témoin (Tableau 2). Le test statistique ANOVA 1 a montré qu'il existe une différence significative entre les biomasses racinaires $(p<0,05)$. Le vermicompost $(45,63 \pm 1,81$ $\left.\mathrm{kg} \mathrm{ha}^{-1}\right)$ a donné la plus importante biomasse racinaire, suivi dans l'ordre décroissant de biomasse par le compost et le sol témoin. Le nombre de grains de maïs par plante présente le même pattern que la biomasse 
épigée ; le vermicompost $(380,1 \pm 12,9)$ et le compost d'ordures ménagères associées aux balles de riz $(370,7 \pm 9,2)$ ont présenté les nombres de grains par plante les plus élevés (Anova $1 ; p<0,05$ ) tandis que ce nombre est plus petit sur le sol témoin (Tableau 2). Le rendement exprimé en biomasse des grains par hectare sur les parcelles amendées au vermicompost

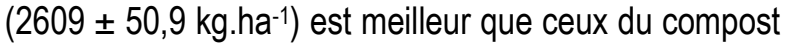
et sol de jachère. Le test statistique ANOVA 1 a montré qu'il y a une différence significative $(p<0,05)$ entre les trois rendements avec le sol témoin qui a produit la plus faible biomasse des grains à l'hectare (Tableau 2).

Tableau 2 : Paramètres de production et rendement du maïs

\begin{tabular}{l|c|c|c|c}
\hline \multirow{2}{*}{ Traitements } & \multicolumn{4}{|c}{ Paramètres de production et rendement } \\
\cline { 2 - 5 } & $\begin{array}{c}\text { Biomasse épigée } \\
\left(\mathrm{kg} \cdot \mathrm{ha}^{-1}\right)\end{array}$ & $\begin{array}{c}\text { Biomasse racinaire } \\
\left(\mathrm{kg}^{-} \mathrm{ha}^{-1}\right)\end{array}$ & $\begin{array}{c}\text { Nombre de } \\
\text { Grains/Plante }\end{array}$ & $\begin{array}{c}\text { Biomasse des grains } \\
\left(\mathrm{kg} \cdot \mathrm{ha}^{-1}\right)\end{array}$ \\
\hline C.OM+br & $66,67 \pm 3,1^{\mathrm{b}}$ & $29,67 \pm 1,8 \mathrm{~b}$ & $370,7 \pm 9,2^{\mathrm{b}}$ & $2108,2 \pm 58,7^{\mathrm{b}}$ \\
VC. OM+br & $76,1 \pm 2,6^{\mathrm{b}}$ & $45,63 \pm 1,81^{\mathrm{c}}$ & $380,1 \pm 12,9^{\mathrm{b}}$ & $2609 \pm 50,9^{\mathrm{c}}$ \\
Sol de jachère & $50,17 \pm 3,40^{\mathrm{a}}$ & $20,21 \pm 1,04^{\mathrm{a}}$ & $300,9 \pm 10,8^{\mathrm{a}}$ & $1810,2 \pm 50,2^{\mathrm{a}}$ \\
\hline$p$ (Anova) & 0,01 & 0,001 & 0,024 & 0,027 \\
\hline
\end{tabular}

$\mathrm{C} . \mathrm{OM}+\mathrm{br}$ : Compost d'Ordures Ménagères associées aux balles de riz, $\mathrm{VC} . \mathrm{OM}+\mathrm{br}$ : Vermicompost d'Ordures Ménagères associées aux balles de riz. Pour un paramètres considéré, les valeurs suivies de lettres différentes sont significativement différentes (Anova 1, $p=0,05$ ).

\section{DISCUSSION}

Les composts produits ont une importante teneur en éléments nutritifs ( $N, P$ et $K$ ), mais les taux en $\mathrm{MO}$ et en éléments majeurs sont plus importants dans le vermicompost que dans le compost d'ordures ménagères. Ce résultat montre que les individus de l'espèce Eudrilus eugeniae ont une grande capacité de minéralisation de la matière organique. Les fortes teneurs en éléments nutritifs disponibles dans les vermicomposts par comparaison aux composts ont également été rapportées par (Bagari \& Biradar, 2017). Ceci serait lié à l'activité de la flore microbienne présente dans les intestins des vers de terre impliquée dans le processus de minéralisation. Laquelle activité de minéralisation est également responsable des faibles teneurs en éléments traces métalliques dans les vermicomposts. En effet, les vers de terre Eudrilus eugeniae ont la capacité de stocker de fortes concentrations de métaux lourds sous les formes non toxiques au cours du transit intestinal des aliments (Morgan \& Morgan, 1998 ; Coulibaly et al., 2014). Ils réduisent ainsi les effets toxiques des métaux lourds superflus en les utilisant pour leur métabolisme physiologique. Les faibles teneurs des vermicomposts en métaux lourds sont également attribuées à la rapide reproduction des vers de terre dans le vermicompost, engendrant ainsi une ingestion plus importante de matière organique par les individus juvéniles et une plus grande accumulation des métaux dans leurs organismes (Coulibaly et al., 2014).

L'application du vermicompost d'ordures ménagères associées aux balles de riz a eu un effet significatif sur le diamètre au collet (à partir du 30 $\mathrm{e} \mathrm{JAS}$ ) ; la hauteur des plants de maïs (à partir du 45 $\mathrm{JAS}$ ) et la longueur des feuilles (à partir du 30 e JAS). L'apport du vermicompost a donc favorisé une libération continue des éléments nutritifs dans le sol et une bonne nutrition des plantes, induisant ainsi une bonne croissance par comparaison aux plants de maïs cultivés sur le compost et le sol sans amendement (Toundou et al., 2014). Ces résultats sont confortés par Coulibaly et al. (2016) qui ont montré que le vermicompost de la fiente de volaille favorise la croissance en hauteur et foliaire de l'espèce Lagenaria siceraria. En effet, les vers de terre Eudrilus eugeniae par la digestion des résidus organiques influencent une plus grande disponibilité des éléments minéraux dans le vermicompost. Au cours du vermicompostage, le transit des déchets à travers le tube digestif des vers de terre a permis une meilleure minéralisation de ceux-ci et par conséquent une augmentation de la teneur des nutriments dans les déjections (turricules) du ver Eudrilus eugeniae (Ndegwa \& Thompson, 2001). Aussi, le mucus sécrété par les vers de terre au cours du vermicompostage augmente la teneur du substrat en azote. Laquelle teneur peut influencer favorablement les paramètres de croissance cités ci-dessus. De manière générale, le vermicompost influence positivement la production et le rendement du maïs. L'utilisation du vermicompost a permis une amélioration significative de la biomasse racinaire et du rendement (biomasse des grains de maïs par hectare) comparativement aux composts simples et au sol non amendé. Nos résultats sont en 
adéquation avec ceux de l'IRD (2015) qui ont montré que le vermicompost favorise la libération rapide des éléments nutritifs dans le sol pour les cultures. Les vermicomposts améliorent les paramètres agronomiques des cultures. Cette amélioration est due d'une part, à l'amélioration des qualités physiques (structure, porosité) et chimiques (teneur en azote et oligoéléments) des sols (Castaldi et al., 2004), et

\section{CONCLUSION}

L'objectif général de notre étude était d'étudier l'impact du vermicompost d'ordures ménagères associées aux balles de riz sur la croissance et le rendement du maïs. $\mathrm{Au}$ bout de 105 jours de compostage, les analyses chimiques du vermicompost et du compost ont révélées une grande variabilité par rapport au sol témoin. En outre, la longueur de la plus grande feuille, la taille moyenne, le diamètre au collet et la biomasse racinaire de maïs du traitement à base de vermicomposts sont plus élevés que ceux du compost et du témoin. Le vermicompost a donné le meilleur rendement en termes

\section{REFERENCES}

Anderson JM. and Ingram J. 1993. Tropical soil biology and fertility : a handbook o methods. CABI, Oxford. $171 \mathrm{p}$.

Bagari G. And Biradar PM. 2017. Analysis of compost and vermicompost produced by the epigeic earthworm, Eudriluseugeniae out of different organic wastes. International journal of currentresearch9 (7) : 53875-53879.

Brou YT. 2005. Climat, mutation socio-économique et paysage en côte d'ivoire. Mémoire de synthèse des activités scientifique. Habilitation à Diriger des recherches, Université des Sciences et Technologie de Lille (France), 212p.

Castaldi P, Garau G, Melis P. 2004. Influence of compost from sea weeds on heavy metal dynamics in the soil-plant system. Fresenius Environment Bulletin 13 : 1322-1328.

Chaichi W, Djazouli Z, Djemai I, Abdelkader S, Ribera I, Nancé J. 2017. Stimulation des défenses naturelles par l'application d'un Lombricompost: Effet sur les paramètres populationnels d'Aphis fabae scop. (Homoptera: Aphididae) et la qualité Phytochimique de la fève. Lebanese Science Journal 18(1) : 81-97.

Coulibaly SS, Edoukou FE, Kouassi KI, Barsan N, Nedeff V, Zoro Bi A. 2018. Vermicompost d'autre part, à la présence d'une microflore tellurique abondante et diversifiée qui favorise la minéralisation de la matière organique (Gomez et al., 2006). De même, Hien et al. (2018) ont montré que l'apport de vermicompost améliore les propriétés physicochimiques du sol, entrainant ainsi l'augmentation de sa productivité.

de biomasse des grains à l'hectare. A l'échelle de cet essai, l'utilisation du vermicompost se présente comme une alternative prometteuse à la gestion durable de la fertilité des sols et respectueuse de l'environnement. Sa production et son utilisation comme amendement permettent non seulement d'assainir l'environnement par le récyclage des ordures ménagères polluantes, mais aussi de produire un vermicompost riche en éléments fertilisants qui permet de soutenir la fertilité déclinante des sols pour une amélioration des productions agricoles.

utilisation : A way to food security in rural area. Heliyon 4e01104. doi : 10.1016/j.heliyon.2018.e01104.

Coulibaly SS, Kouassi KI, Tondoh JE, Zoro Bi A. 2014. Influence of the population size of the earthworm Eudrilus eugeniae on the heavy metal content reduction during vermicomposting of animal wastes. Applied Science Report 7(2) : 96-103.

Coulibaly SS, Tondoh EJ, Kouassi KI, Barsan N. 2016. Vermicomposts improve yields and seeds quality of Lagenaria siceraria in Côte d'Ivoire. International Journal Agronomie Agricultural Research 8: 26-37.

Dabin B, Leneuf N. Riou G. 1960. Carte pédologique de la Côte d'Ivoire au 1/2.000.000. Notice explicative. ORSTOM, $39 \mathrm{p}$.

Deka H, Deka S, Baruah CK, Sarma SN. 2011. Vermicomposting of distillation waste of citronella plant (Cymbopogon winterianus Jowitt.) employing Eudrilus eugeniae. Bioresource Technology 102 : 6944-6950.

FAO. 2020. Agricultural production, crop primary data base. Rome. http://www.fao.org/faostat Consulted on 04/2020.

FAO. 2015. Perspectives pour l'environnement. $9 \mathrm{p}$.

Guéi AM, N'Dri JK, Zro FG, Bakayoko S, Tondoh JE. 2019. Relationships between soil morpho- 
chemical parameters and earthworm community attributes in tropical agroecosystems in the center-west region of Ivory Coast, Africa. Tropical Ecology 60: 209-218.

Guéi AM, Zro FG, Soro D, Kouassi PK. 2020. Étude de l'effet de différentes doses de bouse fraiche de bovin sur la productivité d'un sol sableux utilisé en maraîchage à Daloa, Centre-ouest de la Côte d'Ivoire.Afrique Science 16(1) : 92-105.

Gomez E, Ferreras L, Toresani S. 2006. Soil bacterial functional diversity as influenced by organic amendment application. Bioresource Technologie 97 : 1484-1489.

Haro H, Semdé K, Bahadio K. 2020. Native arbuscular mycorrhizal inoculation of corn (Zea mays L.) cultivated in Burkina Faso. Journal of Applied Biosciences 149: 15291-15296.

Hien V, Ehouman NM, Touré M. Tiho S. 2018. Effets du vermicompost à base de coques de cacao et de graminées sur quelques paramètres agronomiques de la tomate du concombre et du chouà Yamoussoukro. Journal of Applied Biosciences126:12707-12716.

IRD. 2015. Restauration de la productivité des sols tropicaux et méditerranéens Contribution à l'agroécologie. Institut De Recherche Pour Le Développement Montpellier. 547 p.

Jones CG, Lawton J H, Shachk M. 1994. Organisms as ecosystem engineers. Oikos 69: $373-386$.

Morgan JE. and Morgan AJ. 1998. The distribution and intracellular compartmentation of metals in the endogeic earthworm Apporectodea calignosa sampled from and unpolluted and metalcontaminated site. Environmental Pollution 99 : 167-175.

Murphy J. Riley JP.1962. A modified single solution method for the determination of phosphate in natural waters. Analysis Chemical Acta 27 : 31-36.

Ndegwa PM. and Thompson SA. 2001. Integrating composting and vermicomposting of the treatment and bioconversion of biosolids. Bioresource Technologie76 : 107-112.

RGPH. 2014. Recensement Général de la Population et de l'Habitat, Côte d'Ivoire, Rapportd'exécution et de présentation des principaux résultats, $49 p$.

Rekha GS, Kaleena PK, Elumalai D, · Srikumaran MP, Maheswari VN. 2018. Effects of vermicompost and plant growth enhancers on the exo-morphological features of Capsicum annum (Linn.) Hepper. International Journal of Recycling of Organic Waste in Agriculture 7:83-88.

Suthar S. 2007. Influence of different food sources on growth and reproduction performance of composting épigées: Eudrilus eugeniae, Perionyx excavatus and Perionyx sansibaricus. Applied Ecology and Environmental Research 5 (2) : 79-92.

Tondoh EJ, Dimobe K, Guéi AM, Adahé L, Baidal L, N'Dri JK, Forkuor G. 2019. Soil Health Changes Over a 25-Year Chronosequence from Forest to Plantation in Rubber Tree (Hevea brasiliensis) Landscapes in Southern Cote d'Ivoire: Do Earthworms Play Role? Frontiers in Environmental Science 7: 19-73.

Toundou O, Tozo K, Amouzouvi KAA, Lankondjoa K, Tchangbedji G, Kili K. Gnon B. 2014. Effets de la biomasse et du compost de Cassia occidentalis $L$. sur la croissance en hauteur, le rendement du maïs (Zea mays L.) et la teneur en NPK d'un sol dégradé en station expérimentale. European Scientifique Journal 10 (3) : 294-308.

Walkley A. and Black IA. 1934. An examination of the Degtjareff method for determining soil organic matter, and a proposed modification of the chromic acid titration method. Soil Science 34 : 29-38. 